\title{
MANIFOLDS WITH FINITE FIRST HOMOLOGY AS CODIMENSION 2 FIBRATORS
}

\author{
ROBERT J. DAVERMAN
}

(Communicated by James E. West)

\begin{abstract}
Given a map $f: M \rightarrow B$ defined on an orientable $(n+2)$-manifold with all point inverses having the homotopy type of a specified closed $n$-manifold $N$, we seek to catalog the manifolds $N$ for which $f$ is always an approximate fibration. Assuming $H_{1}(N)$ finite, we deduce that the cohomology sheaf of $f$ is locally constant provided $N$ admits no self-map of degree $d>1$ when $H_{1}(N)$ has a cyclic subgroup of order $d$. For manifolds $N$ possessing additional features, we achieve the approximate fibration conclusion.
\end{abstract}

\section{INTRODUCTION}

Codimension 2 fibrators, a notion introduced in [Da], afford quick recognition of certain maps $p: M \rightarrow B$ as approximate fibrations. This, in turn, gives rise to reasonably computable structural information, based on a long exact homotopy sequence for approximate fibrations, developed by Coram and Duvall [CD1], analogous to the classical one for fibrations. Known codimension 2 fibrators include all simply connected closed manifolds, closed 2-manifolds of negative Euler characteristic, and $P^{n}$ (real projective $n$-space); nonfibrators in codimension 2 include all closed manifolds admitting a fixed point free cyclic action having orbit space homotopy equivalent to itself [Da].

A closed, connected manifold $N^{n}$ is called a codimension 2 (orientable) fibrator if whenever $f: M \rightarrow B$ is a proper closed map defined on an (orientable) $(n+2)$-manifold and each $f^{-1}(b)$ is a compact ANR with the homotopy type of $N^{n}, f$ is an approximate fibration (meaning, $f$ satisfies the approximate homotopy lifting property; see [CD1]). In this setting, the upper semicontinuous (henceforth, abbreviated as usc) decomposition $\left\{f^{-1}(b): b \in B\right\}$ of $M$ induced by $f$ is called $N^{n}$-like. Instead of the ANR hypothesis, one could just as well require all $f^{-1}(b)$ in an $N^{n}$-like decomposition to be compact sets shape equivalent to $N^{n}$.

Received by the editors February 8, 1989 and, in revised form, September 5, 1989.

1980 Mathematics Subject Classification (1985 Revision). Primary 55R65, 57N15, 57N65; Secondary $55 \mathrm{M} 25,57 \mathrm{~N} 12,54 \mathrm{~B} 15$.

Key words and phrases. Approximate fibration, approximate lifting, continuity set, codimension 2 fibrator, geometric structure, Hopfain group, mapping degree.

Research supported in part by a grant from the National Science Foundation. 
By the degree of a map between closed, connected, orientable $n$-manifolds, we mean more precisely the absolute degree; namely, the nonnegative integer determining the induced homomorphism's effect on $n$th (integral) homology, up to sign. Let $N^{n}$ be a closed orientable $n$-manifold and $G$ an $N^{n}$-like decomposition of an orientable $(n+2)$-manifold $M$. Then the decomposition space $B=M / G$ is a 2-manifold [DW]. Further analysis depends on the concept of the continuity set $C \subset B$ of the decomposition map $p: M \rightarrow B$, which we briefly describe now in terms of degree: each $g \in G$ has a neighborhood $U_{g}$ equipped with a retraction $R_{g}: U_{g} \rightarrow g$, and $p(g) \in C$ iff $R_{g}$ restricts to a degree 1 map $g^{\prime} \rightarrow g$ for all $g^{\prime} \in G$ sufficiently close to $g$. By [DW] $C$ contains all but a locally finite subset of $B$.

Recall that a group $\Gamma$ is Hopfian if every epimorphism $\Gamma \rightarrow \Gamma$ is necessarily $1-1$.

In lieu of additional review, we restate two basic facts from [Da] in a form readily applicable here.

Proposition AF. Suppose $N^{n}$ is a closed orientable n-manifold such that either $\pi_{1}\left(N^{n}\right)$ is finite or it is Hopfian and $N^{n}$ is aspherical, and suppose $G$ is an $N^{n}$. like usc decomposition of an orientable $(n+2)$-manifold $M$. Then the decomposition map $p: M \rightarrow B$ restricts to an approximate fibration $p \mid p^{-1}(C): p^{-1}(C) \rightarrow$ $C$ over the continuity set $C$ of $p$.

We close by outlining the strategy. Under the hypothesis that $H_{1}\left(N^{n}\right)$ is finite, we seek to prove that the continuity sets $C$ of $p: M \rightarrow B$ associated with various $N^{n}$-like decompositions satisfy $C=B$. The homological finiteness condition alone does not force $N^{n}$ to be a codimension 2 fibrator $-P^{2 k+1} \# P^{2 k+1}$ is a counterexample [Da]. Nevertheless, then all $R_{g}$ restrict to maps $g^{\prime} \rightarrow g$ with nonzero degree, useful data in itself, and the first result in the next section limits the possible degrees $d$ to the orders of cyclic subgroups of $H_{1}\left(N^{n}\right)$. Of course, $C=B$ when $d=1$ is the only possibility. Finally, we impose further conditions on $N^{n}$, like those in the hypothesis of Proposition AF, to detect approximate fibrations.

\section{MANIFOLDS WITH FINITE FIRST HOMOLOGY}

Proposition 1. Suppose $N^{n}$ is a closed, orientable n-manifold whose first (integral) homology group is finite. Suppose $M$ is any orientable $(n+2)$-manifold, $G$ is an $N^{n}$-like usc decomposition of $M, g_{0} \in G$, and $R: U \rightarrow g_{0}$ is a retraction defined on a neighborhood $U$ of $g_{0}$. Then there exists an integer $d \geq 1$ such that

(1) $g_{0}$ has a neighborhood $U^{\prime} \subset U$ where, for all $g \in G$ in $U^{\prime}, g \neq g_{0}$, $R \mid g: g \rightarrow g_{0}$ has absolute degree $d$;

(2) $H_{1}\left(N^{n}\right)$ contains a cyclic subgroup of order $d$. 
Proof. At the core of the argument, which mostly involves algebraic diagramchasing, is the simplification stemming from the relationships $H_{n-1}\left(N^{n}\right) \cong$ $H^{1}\left(N^{n}\right) \cong 0$ insured by duality and universal coefficients.

Consider the decomposition map $p: M \rightarrow B=M / G$. In this setting $B$ is a 2-manifold [DW]. Restricting $M$, we reduce to the situation where $B$ is an open 2-cell containing $b_{0}=p\left(g_{0}\right)$ and $p: M \rightarrow B$ is an approximate fibration over $B \backslash b_{0}$.

Application of approximate lifting over $B \backslash b_{0}$ shows $M$ deformation retracts to $g_{0}$, with $R: M \rightarrow g_{0}$ being the end of the deformation. Accordingly, $R_{*}: H_{*}(M) \rightarrow H_{*}\left(g_{0}\right)$ is an isomorphism. From the exact sequence of the pair $\left(M, M \backslash g_{0}\right)$,

$$
0 \cong H_{n+2}(M) \rightarrow H_{n+2}\left(M, M \backslash g_{0}\right) \rightarrow H_{n+1}\left(M \backslash g_{0}\right) \rightarrow H_{n+1}(M) \cong 0,
$$

yielding $H_{n+1}\left(M \backslash g_{0}\right) \cong Z$, and

$$
0 \cong H^{1}\left(g_{0}\right) \cong H_{n+1}\left(M, M \backslash g_{0}\right) \rightarrow H_{n}\left(M \backslash g_{0}\right) \rightarrow H_{n}(M) \cong Z .
$$

Finiteness of $H_{1}\left(N^{n}\right)$ is crucial to proving $H_{n}\left(M \backslash g_{0}\right)$ is the (isomorphic) image of $H_{n}(g)$ for any $g \in G \backslash\left\{g_{0}\right\}$. Express $M \backslash g_{0}$ as the union of $G$ saturated open sets $V, V^{\prime}$ whose intersection consists of components $W, W^{\prime}$, where $p(V), p\left(V^{\prime}\right), p(W), p\left(W^{\prime}\right)$ are all 2-cells and $g \subset W$. Since

$$
\begin{gathered}
H_{n}(V) \cong H_{n}(W) \cong H_{n}(g) \cong Z \cong H_{n}\left(V^{\prime}\right) \cong H_{n}\left(W^{\prime}\right), \\
H_{n-1}(W) \cong H_{n-1}(g) \cong 0 \cong H_{n-1}\left(W^{\prime}\right),
\end{gathered}
$$

the Mayer-Vietoris sequence for the triad $\left(M \backslash g_{0}, V, V^{\prime}\right)$ shows

$$
0 \rightarrow Z \cong H_{n+1}\left(M \backslash g_{0}\right) \rightarrow Z \oplus Z \stackrel{\psi_{*}}{\longrightarrow} Z \oplus Z \rightarrow H_{n}\left(M \backslash g_{0}\right) \rightarrow 0 .
$$

Focus on the homomorphism $H_{n}(W) \oplus H_{n}\left(W^{\prime}\right) \rightarrow H_{n}(V) \oplus H_{n}\left(V^{\prime}\right)$ giving rise to the homomorphism $\psi_{*}: Z \oplus Z \rightarrow Z \oplus Z$ in $(\zeta)$, and arrange notation to make $\psi_{*}(\langle s, t\rangle)=\langle s+t, s \pm t\rangle$. Sequence $(\zeta)$ shows $\operatorname{ker} \psi_{*} \neq 0$, so $\psi_{*}(\langle s, t\rangle)=\langle s+t, s+t\rangle$ and, thus, $H_{n}\left(M \backslash g_{0}\right) \cong(Z \oplus Z) / \operatorname{im}\left(\psi_{*}\right)$ corresponds to $H_{n}(V) \oplus 0 \cong H_{n}(g) \oplus 0$.

Now examine the diagram

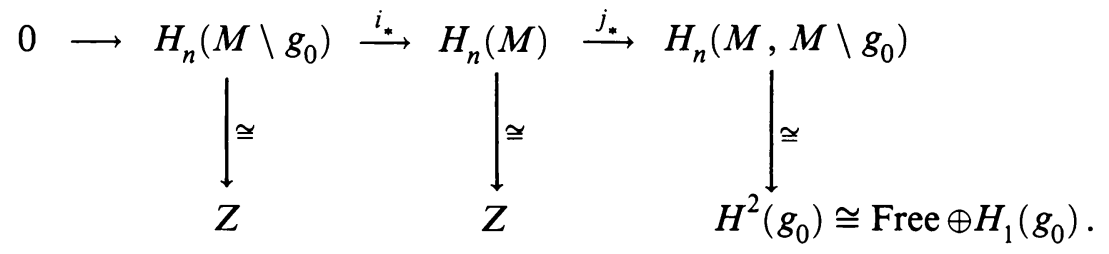

Up to sign, $i_{*}$ amounts to multiplication by an integer $d \geq 1$, and $j_{*}$ injects the cokernel, a cyclic subgroup of order $d$, in $H_{1}\left(g_{0}\right) \cong H_{1}\left(N^{n}\right)$. Finally, $R \mid g: g \rightarrow g_{0}$ has degree $d$, since the induced homomorphism on $n$th homology can be expressed as the composition of isomorphisms with $i_{*}$.

Corollary 2. Suppose $N^{n}$ is a closed, orientable, n-manifold such that $H_{1}\left(N^{n}\right)$ is finite and whenever $H_{1}\left(N^{n}\right)$ contains a cyclic subgroup of order $d>1$, then 
$N^{n}$ admits no self-map of degree $d$. Suppose $G$ is an $N^{n}$-like usc decomposition of an orientable $(n+2)$-manifold $M$. Then the (integral) cohomology sheaf of the decomposition map $f: M \rightarrow B=M / G$ is locally constant.

Proof. Clearly the maps $R \mid g: g_{0}$ induce isomorphisms on $n$th homology. By Poincare duality and naturality of cap products, they have the same effect on homology groups at all levels (application of 5.6 .16 from [Sp, p. 254] reveals that these $(R \mid g)^{*}$ provide epimorphisms and hence isomorphisms between $(n-q)$ th homology groups). Duality implies these $(R \mid g)^{*}$ are cohomology isomorphisms in all dimensions, from which the corollary follows.

Propositions 1 and AF combine as:

Theorem 3. Suppose $N^{n}$ is a closed, orientable, n-manifold for which either $\pi_{1}\left(N^{n}\right)$ is finite or $\pi_{1}\left(N^{n}\right)$ is Hopfian, $H_{1}\left(N^{n}\right)$ is finite, and $N^{n}$ is aspherical. Suppose further that if $H_{1}\left(N^{n}\right)$ contains a cyclic subgroup of order $d>1$ then $N^{n}$ admits no self-map of degree $d$. Then $N^{n}$ is a codimension 2 orientable fibrator.

Corollary 4. If $\Gamma$ is a finite group admitting no homomorphisms $\psi: \Gamma \rightarrow \Gamma$ with index $[\Gamma, \psi(\Gamma)]>1$ equal to the order of a cyclic subgroup of the abelianization, $\Gamma / \operatorname{comm}(\Gamma)$, then every orientable manifold $N$ with $\pi_{1}(N) \cong \Gamma$ is an orientable codimension 2 fibrator.

Proof. Given $N$ with fundamental group $\Gamma$, consider any map $f: N \rightarrow N$ of degree $d>1$, where $d$ is the order of a cyclic subgroup of $H_{1}(N) \cong$ $\Gamma / \operatorname{comm}(\Gamma)$. Suppose $f_{\#}(\Gamma) \neq \Gamma$. Specify a covering space $q: N^{*} \rightarrow N$ corresponding to $f_{\#}(\Gamma)$ and a lift $f^{*}: N \rightarrow N^{*}$ of $f$. Since

$$
d=\operatorname{deg}(f)=\operatorname{deg}(q) \cdot \operatorname{deg}\left(f^{*}\right),
$$

we see that $\left[\Gamma, f_{\#}(\Gamma)\right]=\operatorname{deg}(q)$ divides $d$, implying the existence of another cyclic subgroup in $H_{1}(N)$ of order $\left[\Gamma, f_{\#}(\Gamma)\right]$, contrary to hypothesis. Thus, all degree $d$ maps between copies of $N$ encountered in our setting induce $\pi_{1}$ isomorphisms, in which case we can imitate the proof of [Da, Proposition 2.6] to conclude $N$ is a codimension 2 fibrator.

For examples, such familiar objects as the symmetric group $\mathscr{S}_{m}$ on $m>4$ symbols, the nonabelian group of order 21 , and the dihedral groups

$$
D_{2^{m} \cdot(2 k+1)}=\left\langle x, y \mid x^{2^{m}}=1=y^{2 k+1}, x y x^{-1}=y^{-1}\right\rangle
$$

all satisfy the hypothesis of Corollary 4 . Inspecting the third example more closely, we see it abelianizes to the cyclic group of order $2^{m}$ and any subgroup $H$ of index $2^{s}$ is cyclic (because $y$ commutes with $x^{2}$ ) of order divisible by $2 k+1$, but obviously then this dihedral group does not surject to $H$.

Finite abelian groups are not amenable to this analysis. 


\section{Applications to 3-MANifolds}

We point out another fact closely related to Proposition AF.

Proposition 5. Suppose $N^{n}$ is a closed orientable n-manifold, $n \in\{3,4\}$, with $\pi_{1}\left(N^{n}\right)$ Hopfian and suppose $G$ is an $N^{n}$-like usc decomposition of an orientable $(n+2)$-manifold $M$. Then the decomposition map $p: M \rightarrow B$ restricts to an approximate fibration $p \mid p^{-1}(C)$ over the continuity set $C$ of $p$.

Proof. Hausmann [Ha, Proposition 1(b)] establishes that, over $C$, the retractions $R_{g}: U_{g} \rightarrow g$ restrict to homotopy equivalences $g^{\prime} \rightarrow g$ for $g^{\prime} \in G$ sufficiently close to $g$. In the context at hand this property yields the conclusion [CD2, Corollary 3.4].

Conceivably the class of manifolds $N^{3}$ described below is all-inclusive. Under this hypothesis Hempel [He2, Corollary 1.2] proves $\pi_{1}\left(N^{3}\right)$ is Hopfian (also see $[\mathrm{He} 1]$ for the revelant definition).

Corollary 6. Suppose $N^{3}$ is a closed, orientable 3-manifold whose prime factors are either virtually Haken or else have finite or cyclic fundamental groups, and suppose $G$ is an $N^{3}$-like usc decomposition of an orientable 5-manifold $M$. Then the decomposition map $p: M \rightarrow B$ restricts to an approximate fibration over its continuity set.

Corollary 7. Every closed hyperbolic 3-manifold with finite first homology is a codimension 2 fibrator.

Proof. According to Gromov [Gr, p. 147], there is no degree $d>1$ self-map on a closed hyperbolic manifold. (Lemma 4.1 of [He2] certifies the Hopfian part needed in Proposition 5.)

The conclusion of Corollary 7 might be valid without the $H_{1}$-finiteness condition.

Let $L^{3}$ denote the unique closed 3-manifold having Euclidean structure and finite first homology (in other words, $L^{3}$ does not fiber over $S^{1}$ ). It is the orientable Seifert bundle over $P^{2}$ with Euclidean structure appearing in Scott's list [Sc, p. 446]. Standard calculations from the associated Seifert bundle data yield $H_{1}\left(L^{3}\right) \cong Z_{4} \oplus Z_{4}$.

Lemma 8. There is no map $L^{3} \rightarrow L^{3}$ of degree 2 or 4 .

Proof. Suppose $f: L^{3} \rightarrow L^{3}$ is a map of degree 2 or 4 . Keep in mind that $f_{\#}\left(\pi_{1}\left(L^{3}\right)\right) \neq \pi_{1}\left(L^{3}\right)$ for otherwise $f_{\#}$ would be an isomorphism and, as a selfmap on a $K(\pi, 1)$ space, $f$ would be a homotopy equivalence and, therefore, have degree 1 . As in the proof of Corollary 4, take a (non-trivial) covering $q: N^{*} \rightarrow L^{3}$ corresponding to $f_{\#}\left(\pi_{1}\left(L^{3}\right)\right)$ and lift $f$ to $f^{*}: L^{3} \rightarrow N^{*}$. Significant features are: $f^{*}$ induces an epimorphism at the $H_{1}$ level (of course, on $\pi_{1}$ as well), and $q$ has degree 2 or 4 .

We can regard $L^{3}$ as the result of identifying two copies $T, T^{\prime}$ of the orientable twisted $I$-bundle over a Klein bottle via the homeomorphism $\theta$ on 
the boundary torus interchanging the $S^{1}$-factors, in some preferred parameterization. (Scott [Sc, p. 448] relates that closed Euclidean 3-manifolds arise either as torus or Klein bottles bundles over $S^{1}$ or as the union of two twisted $I$-bundles, and the finiteness of $H_{1}\left(L^{3}\right)$ precludes $L^{3}$ from being among the former.) Thus, $N^{*}$ is a union of finite-sheeted covers of $T, T^{\prime}$, which necessarily are either $I$-bundles over the torus or homeormorphic lifts of $T$ and $T^{\prime}$ themselves.

Suppose $q$ is 2-sheeted. Then $N^{*}$ could be a torus bundle over $S^{1}$ (with monodromy $\theta$ ); this occurs iff both twisted $I$-bundles lift to $S^{1} \times S^{1} \times I$. Otherwise $N^{*}$ is another union of twisted $I$-bundles over a Klein bottle. The latter occurs when (precisely) one of $T, T^{\prime}$ is covered by two copies of itself and $N^{*}$ coincides with their union plus a boundary collar); a check of the various lifts shows the attaching homeomorphism to be the identity. Neither case can arise in the present context, since each requires $H_{1}\left(N^{*}\right)$ to be infinite.

Moreover, $q$ cannot be 4-sheeted either, for it follows easily that $q$ would factor through a 2-sheeted cover, again implying $H_{1}\left(N^{*}\right)$ is infinite.

Corollary 9. $L^{3}$ is an orientable codimension 2 fibrator.

Among closed 3-manifolds having Euclidean geometric structure, $L^{3}$ is the solitary example satisfying the conclusion of Corollary 9. Like all Euclidean manifolds, $L^{3}$ covers itself-in fact, it does so $k$ times for every odd integer $k$ (e.g., the cover corresponding to the subgroup generated by $x^{k}, y^{2}$ in the presentation for $\pi_{1}\left(L^{3}\right)$ as

$$
\left\langle x, y \mid x y^{2}=y^{-2} x, y x^{2}=x^{-2} y\right\rangle,
$$

where $x, y^{2}$ generate the contribution of one of these twisted $I$-bundles while $y, x^{2}$ generate the other), but none of these self-covers is regular cyclic (otherwise $L^{3}$ would not be a codimension 2 fibrator [Da, Theorem 4.2]).

Remarks. Both $S^{3}$ and $P^{3}$ are known to be codimension 2 fibrators (cf. [Da, Theorems 2.1 and 6.1]). The techniques developed here show that additional manifolds with $S^{3}$ geometric structure share the property; namely, those with fundamental group equal to a dihedral group $D_{2^{m} \cdot(2 k+1)}(m>1)$. We do not know whether the same holds for Lens spaces $L(p, q)$ when $p>2$. Analysis similar to that of Lemma 8 reveals that many Siefert fibered 3-manifolds with other geometric structures and finite first homology are codimension 2 fibrators.

\section{REFERENCES}

[CD1] D. S. Coram and P. F. Duvall, Approximate fibrations, Rocky Mountain J. Math. 7 (1977), 275-288.

[CD2] _- Approximate fibrations and a movability condition for maps, Pacific J. Math. 72 (1977), 41-56.

[Da] R. J. Daverman, Submanifold decompositions that yield approximate fibrations, Topology Appl. 33 (1989), 173-184. 
[DW] R. J. Daverman and J. J. Walsh, Decompositions into codimension two manifolds, Trans. Amer. Math. Soc. 288 (1985), 273-291.

[Gr] M. Gromov, Hyperbolic groups, Essays in Group Theory (S. M. Gersten, ed.), Math. Sciences Research Inst. Publ., Springer-Verlag, New York and Berlin, 1987, pp. 75-263.

[Ha] J.-C. Hausmann, Geometric Hopfian and non-Hopfian situations, Geometry and Topology, (C. McCrory and T. Shifrin, eds.), Lecture Notes in Pure Appl. Math., vol. 105, Marcel Dekker, New York, 1987, pp. 157-166.

[He1] J. Hempel, 3-Manifolds, Ann. of Math. Stud., No. 86, Princeton Univ. Press, Princeton, NJ, 1976.

[He2] _ Residual finiteness for 3-manifolds, Combinatorial Group Theory and Topology (S. M. Gersten and J. R. Stallings, eds.), Ann. of Math. Stud., No. 111, Princeton Univ. Press, 1987, pp. 379-396.

[Sc] G. P. Scott, The geometries of 3-manifolds, Bull. London. Math. Soc. 15 (1983), 401-487.

[Sp] E. H. Spanier, Algebraic topology, McGraw-Hill, New York, 1966.

Department of Mathematics, The University of Tennessee, Knoxville, Tennessee 37996-1300 